\title{
Research on the Interactive Teaching of Yoga and Aerobics
}

\author{
Yanhong Zhang, Junlong Tian, Tengteng Zhang \\ Nanchang Institute of Science \&Technology, Nanchang City, Jiangxi Province, China, 330108
}

Keywords: Yoga, Aerobics, Distinction and Connection, Interactive Teaching

\begin{abstract}
In the modern society, the pursuit of health beauty has become a fashion, and Yoga, Aerobics both have a unique charm, so more and more people are beginning to enjoy the sports. Because the Aerobics and Yoga both have some effects on physical fitness, body shape, mood adjusting and mental stress relief, so they have a strong appeal for the young people who are engaged in the heavy schoolwork. Based on the author's teaching experience, this paper first analyzed the relations between Yoga and Aerobics, then discussed the important differences, finally put forward the method of interactive teaching. The organic combination of the two kinds of sports can reduce the negative impact on students in Aerobics teaching, and play a role in promoting the healthy growth of students.
\end{abstract}

\section{Introduction}

Aerobics and Yoga classes have emerged in a lot of ordinary college physical education courses, and it is usually loved by the girls. However, there are some problems to open the course, for example, due to resource allocation, teaching arrangements and other reasons, most of the female students both like Aerobics and Yoga, but they can only choose one course, so they have to spend time and money to the outside club to learn another course. Therefore, Yoga and Aerobics interactive teaching can not only meet the needs of students to strengthen their body, but also can meet the needs of students' preferences in the teaching of physical education in colleges and universities, so it will be welcomed and loved by the majority of girls in the future curriculum development.

\section{The Relations between Yoga and Aerobics}

Consistency of exercise characteristic. Their exercise characteristics are to shape the beauty of the body and to cultivate a healthy body and mind by completing specific action. The specific exercise process of Yoga is carried out in a certain period of time and a certain space range, and the body's coordination is the main form of expression, showing a variety of actions. Aerobics is also in a certain time and space, without props to carry on one kind of body movement. So it is not difficult to see that the consistency of the characteristics of Yoga and Aerobics.

Expression forms. In the actual movement process, with the music rhythm, the exerciser shows different body movements and different body style, in order to achieve the goal and effect.

Impact on the body. In the actual exercise, whether it is through Yoga or Aerobics exercise, both of them have a certain requirement for the physical strength and coordination degree, can effectively improve the flexibility of the muscles and the strength of the organ function though the the exercise, which has a great effect on improving the physical fitness and physical function of the exerciser. 


\section{The Important Differences between Yoga and Aerobics}

There is an important difference between Yoga and Aerobics in the form of sport. Although Yoga and Aerobics all need music, the musical frequencies of the two sports are quite different. Yoga music is generally inclined to nature and lyric, and elegant, slow are important features of Yoga music. However, the Aerobics music requires a cheerful rhythm, can greatly stimulate the mood of the people, to provide a good rhythm for Aerobics. The important differences between the two kinds of movement in the music cause the difference of the body movement. In the process of Yoga movement, it needs a soothing music to stretch the body, to guide the movement of the body and mind to reach a consensus based on the psychological ideas. As a whole body movement needing for a certain period of time, there is an important difference between Aerobics and Yoga.

There are differences between Yoga and Aerobics in the form of action. In the process of fitness, Yoga exercise main trains breathing rhythm to verge the self stretching and breathing movement. Yoga can fully regulate the movement of the nervous mood to a certain extent, to achieve the purpose of sports fitness. However, Aerobics is a continuous movement, it needs to ensure the strength of the action, the magnitude and intensity of motion during the exercise, which has a positive effect on the body's reaction ability and physical fitness.

The effect of Yoga and Aerobics has their own emphasis. Based on the two different forms of performance, so the two kinds of movement in the final effect is also different. Because the movement tends to be quiet, Yoga has a positive effect on the physical and mental health of the sports, and some psychological problems can be significantly improved. In the process of Aerobics exercise, exercise person's body response ability, jumping ability, heart lung function can be promoted, which can effectively supplement the deficiency of Yoga movement. In the actual motion selection process, exerciser should choose different exercise methods according to their different needs. If you focus on physical and mental health and the spirit of the world, you can choose Yoga exercise, and if you tend to physical exercise, you need to choose Aerobics.

\section{The Interactive Teaching Strategies of Yoga and Aerobics}

Using Yoga to prepare for aerobic exercise. Before the Aerobics, it must be prepared to prevent the occurrence of injuries in the course of the movement. So before the Aerobics sports, we can do some warm up exercises through Yoga to lay a good foundation for the formal development of Aerobics. Traditional sports warm-up activity is jogging, which can not effectively control the psychological status of students, and it is difficult to realize the concentration of thoughts in the process of movement, so that it lays down the hidden danger to the Aerobics movement. Yoga exercise before the warm-up exercises, on the one hand, it can quickly improve the body of the exercise of the excitement, to ensure that the process of Aerobics exercise will not be an accident. On the other hand, it also can improve the students' concentration degree and enter the normal class order as soon as possible, which plays an important part in the teaching quality and safety of Aerobics.

Using Yoga exercise to complete the exercise of flexibility exercises and relaxation exercises. Aerobic exercise has certain requirements for the physical exercise of the body's flexibility, a good flexibility is one of the basic requirements of aerobic exercise. In the flexibility of the exercise process, appropriate use of Yoga exercise can effectively enhance the body's flexibility. Through Yoga, it can effectively improve the tension of the body, change the tensile state of the muscles, during the exercise, listening to soothing, elegant music, the body will be free to stretch, which can be effective to relieve the tension of the students, so as to have a positive effect on the students' 
flexibility. In the daily teaching of Aerobics, the high rhythm and fast frequency of the exercise in a short period of time make students feel tired, if we do not effectively alleviate the fatigue status of the exercise, it will certainly affect the teaching of Aerobics. In the specific classroom teaching of Aerobics, we should take some measures to make a effective combination of Aerobics and Yoga. After a certain time of Aerobics exercise, selecting proper Yoga exercise, in order to achieve the purpose of reducing the burden. The effective combination of Yoga and Aerobics can make the students return to their normal state as soon as possible from the state of aerobic exercise, so that it can reduce the student's excitement and save the student's physical strength.

The key issues of Yoga Yoga in the course of Aerobics teaching. As a traditional sport, Yoga have formed the different factions after years of development, although they are called Yoga, the movement difficulty and movement effect has been significantly differentiated, so choose the right Yoga Aerobics is very important in the process. In the actual Aerobics teaching, teachers should choose the appropriate Yoga action according to the specific situation of the students in the class, try to choose some actions which is not difficult, to ensure students' active status, to lay a good foundation for students to complete the Aerobics class task. In the time to teach Yoga, teachers should guide students to breathe, to ensure the effect of Yoga learning, if they teach the simple action of Yoga and learning, it is difficult to achieve the real purpose of Yoga learning. In the course of Yoga, it should always pay attention to the purpose of Yoga learning, learning just for the Aerobics can not be the main content of Yoga exercise. The Yoga practice before the start of aerobic exercises should pay attention to grasp the time, and the time it takes to do Yoga is not too long. As long as the it reached the basic requirements before the start of Aerobics, we can stop the practice of Yoga, The organic combination of Yoga and aerobic exercise can achieve the effect of exercise in the following exercise,

\section{The Great Significance of Yoga and Aerobics Interactive Teaching.}

Effectively preventing the students from sports loss accidents. Yoga exercises can stretch all parts of the body, meet the requirements of the preparation work before Aerobics, so students can effectively avoid the occurrence of sports injury accidents. As a movement with slower pace, the stretching speed of Yoga is slower for muscles and ligaments. Yoga exercise can gradually elevate the body's excitement, even the students rarely participating in any sports can be also be put into practice as soon as possible, laying a good foundation for the development of Aerobics.

It is helpful to ease students' pressure and relieve the psychological burden. As an effective stress reduction measure, physical exercise plays an important role in the practical application. In the current society, college students are under the pressure of study, employment, and it caused the long-term depression of College Students' mentality and anxiety. However, the Aerobics and Yoga interactive teaching can effectively release students' psychological pressure brought by the Aerobics, alleviate the long-term depression of college students' mental attitude, help students to find a better life, to restore the confidence of the mentality

\section{Conclusion}

Health is the most basic condition for every person to live, and Aerobics has some characteristics of artistic, rhythmic and adaptability, so it is popular among people. The development of Aerobics teaching course can be one of the means to improve students' comprehensive quality. However, positively introducing Yoga training in the practice teaching of Aerobics can strengthen the students' physical ability and their psychological quality. Therefore, the interactive teaching of 
Aerobics and Yoga can greatly improve the teaching effect and quality.

\section{References}

[1] Du Xiru. An experimental study of yoga fitness in Aerobics Teaching [J]. Journal of Guangzhou Sports University, 2006,01:109-112.

[2] Liu Ziting, Xiao Ningning, Wang Shaofeng. An experimental study on the combination of aerobics and yoga skills [J]. Journal of natural science of Harbin Normal University, 2006,06:99-104.

[3] Liu Shumei, Yang Guang. A comparative study of yoga and aerobics exercises on improving flexibility [J]. Exercise, 2014,13:75-76+146.

[4] Li Xiaochan, Wang Zhiguo, Jin Longquan. Exploring the integration of Yoga aerobics course in Colleges and universities [J]. Journal of Ezhou University, 2014,08:99-100. 